It is now sufficient to show that

$$
\left(f_{t}^{*}, \Phi\right)=\int \exp [-i E(k) t] f(k) \Phi(k) d^{3} k \rightarrow 0 .
$$

A change of variables from $k$ to $E$ and two angle variables $\Omega$ gives

$$
\left(f_{t}^{*}, \Phi\right)=\int \exp (-i E t) f(E, \Omega) \Phi(E, \Omega) J d E d \Omega
$$

where $J$ is the Jacobian. By Schwarz's inequality, the product of two square-integrable functions $f$ and $\Phi$ is absolutely integrable with the weight function $J$. The Riemann-Lebesgue lemma then asserts the asymptotic vanishing of the norm $\left\|a_{f t} \psi\right\|$.

The "asymptotic vanishing of destruction operators" was used previously by Coester and Kummel. ${ }^{18}$

${ }^{18}$ F. Coester and H. Kummel, Nuclear Phys. 9, 225 (1959), Appendix II.

\title{
Canonical Variables for General Relativity
}

\author{
R. ARNOWITT* \\ Department of Physics, Syracuse University, Syracuse, New York \\ S. DESER* \\ Department of Physics, Brandeis University, Waltham, Massachusetts \\ AND \\ C. W. MISNER $\dagger$ \\ Universitetets Institut Teoretisk for Fysik, Copenhagen, Denmark
}

(Received October 12, 1959)

\begin{abstract}
The general theory of relativity is cast into normal Hamiltonian form in terms of two pairs of independent conjugate field variables. These variables are explicitly exhibited and obey ordinary Poisson bracket relations. This form is reached by imposing a simple set of coordinate conditions. It is shown that those functionals of the metric used as invariant coordinates do not appear explicitly in the Hamiltonian and momentum densities, so that the standard differential conservation laws hold. The bearing of these results on the quantization problem is discussed.
\end{abstract}

\section{INTRODUCTION}

$\mathbf{I}^{\mathrm{N}}$ $\mathrm{N}$ the program of quantization of general relativity according to the Schwinger action principle it has previously been shown that the classical theory can, in principle, be reduced to canonical form in terms of two pairs of independent, unconstrained canonical variables. ${ }^{1}$ This canonical form has been given explicitly for the linearized theory in I, where the analysis was in complete analogy to the quantization of electromagnetic theory. In II, a general study of the dynamics of the full classical theory led to the exhibition of four unconstrained variables whose specification fully determines the state of the system, but which were not canonical. The precise definition of these variables

\footnotetext{
* Supported in part by a National Science Foundation Research Grant.

$\dagger$ Alfred P. Sloan Research Fellow. On leave from Palmer Physical Laboratory, Princeton University, Princeton, New Jersey.

${ }_{1}$ Two previous papers in this series, R. Arnowitt and S. Deser, Phys. Rev. 113, 745 (1959); and R. Arnowitt, S. Deser, and C. W. Misner, Phys. Rev. 116, 1322 (1959), will be referred to as I and II, respectively. Notation and units are as in II with the exception that $g^{i j}$ here denotes the three dimensional matrix inverse to $g_{i j}$. Natural units are employed: $\kappa=16 \pi \gamma c^{-4}=1, c=1$, where $\gamma$ is the Newtonian gravitational constant. Latin indices run from 1 to 3 , and Greek from 0 to 3 and $x^{0}=t$. Ordinary differentiation is denoted by a comma in a subscript or by the symbol $\partial_{\mu}$.
}

depended upon the specification of the four remaining field variables (the gauge functions) as invariant coordinates. In general, these four field variables will appear explicitly in the Hamiltonian density which arises when the dynamical variables are rearranged into canonical form. Thus, the Hamiltonian density will in general depend upon the coordinates explicitly and hence the system will appear to be nonconservative for a closed system. The remaining problem, as was stressed in II, was thus to achieve canonical form while simultaneously choosing as coordinates a set of gauge variables which will not appear in the resulting Hamiltonian.

This paper exhibits explicitly a simple set of canonical variables and coordinate conditions which solve the above problem.

\section{FORMULATION OF THE PROBLEM}

We begin with the action integral obtained in II when the algebraic constraints have been eliminated. Written in terms of variables appropriate to a $3+1$ dimensional breakup it becomes

$$
I=\int d^{4} x \mathcal{L}
$$


where

$$
\begin{aligned}
\mathscr{L}= & \left(-{ }^{4} g\right)^{\frac{1}{2}}{ }^{4} R=\left({ }^{3} g\right)^{\frac{1}{2}}\left[-g^{i j} \partial_{0} K_{i j}-\partial_{0} K\right. \\
& +N\left({ }^{3} R+K^{2}-K_{i j} K^{i j}\right)-2 \eta^{i}\left(K^{i}{ }_{i}-\delta^{j}{ }_{i} K\right)_{\mid j} \\
& \left.-2\left(N_{, i}-\eta^{j} K_{i j}\right)^{\mid i}\right]
\end{aligned}
$$

$N=\left(-g^{00}\right)^{-\frac{1}{2}}, \quad \eta_{i}=g_{0 i}, \quad K_{i j}=-N \Gamma_{i j}^{0}$ and $K=g^{i j} K_{i j}$. In Eq. (2.2) the symbol "," means covariant differentiation with respect to the three dimensional metric $g_{i j}$. This three-metric and its reciprocal $g^{i j} \equiv{ }^{4} g^{i j}+N^{-2} \eta^{i} \eta^{j}$ are used to raise and lower indices and form the curvature scalar ${ }^{3} R$. The action, being linear in time derivatives, is to be varied independently with respect to $g^{i j}$ and $K_{i j}$ as well as $N$ and $\eta^{i}$ which were seen to play the role of Lagrange multipliers.

The variation of the Lagrangian with respect to $N$ and $\eta^{i}$ gives rise to the four equations not involving time derivatives, i.e., the constraint equations. The variation with respect to $g^{i j}$ and $K_{i j}$ yields the equations involving the time derivatives of these variables. As was seen in II, the twelve $g^{i j}$ and $K_{i j}$ variables can be expressed in terms of three groups of four variables, $u^{\rho}$, $v^{\rho}$, and $w^{\rho}(\rho=1,2,3,4$ is not a tensor index $)$. Inversely, the $u^{\rho}$, $v^{\rho}$, $w^{\rho}$ are functionals of $g^{i j}$ and $K_{i j}$. The $w^{\rho}$ are four variables obtained in terms of $u^{\rho}$, $v^{\rho}$ by solving the constraint equations $\left[w^{\rho}=f^{\rho}\left(u^{\lambda}, v^{\lambda}\right)\right]$; the latter do not of course contain the Lagrange multipliers $N$ and $\eta^{i}$. The $u^{\rho}$ are dynamical variables and the $v^{\rho}$ gauge variables. This nomenclature corresponds to a grouping of the twelve equations involving time derivatives according to the following scheme: the $\partial_{0} v^{\rho}$ equations are to be regarded as equations that determine $N$ and $\eta^{i}$ as functions of $u^{\rho}$, $v^{\rho}$ and $\partial_{0} v^{\rho}$. Consistent with calling the $v^{\rho}$ gauge functions, the time derivatives are not determined dynamically and so the $v^{\rho}$ are arbitrary. The four $\partial_{0} u^{\rho}$ equations represent the first order equations for the two sets of dynamical degrees of freedom of the field. Finally the $\partial_{0} w^{p}$ equations are the Bianchi identities since they guarantee that the constraint equations are maintained in time. An explicit set of $u^{\rho}$, $v^{\rho}$, and $w^{\rho}$ were discussed in II, though this set did not lead to a canonical form of the theory. The criteria which the choice of $u^{\rho}$ and $v^{\rho}$ must satisfy in order to put the theory into canonical form with a conserved Hamiltonian can be obtained by examining the generator. In general, the generator which arises from varying the action at the boundaries, ${ }^{1}$ takes the form

$$
G=\int d^{3} x\left[f_{\rho}(u, v) \delta u^{\rho}+h_{\rho}(u, v) \delta v^{\rho}\right]
$$

when the differential constraint variables, $w^{\rho}$, have been eliminated. No terms of the form $T^{0}{ }_{\mu} \delta x^{\mu}$ appear in Eq. (2.3) since it will be recalled that these $T^{0}{ }_{\mu}$ vanish as a consequence of the constraints. Since we have seen that the $v^{\rho}$ are not determined dynamically they may be used as independent variables in place of coordinates. (This finds an analogy in a procedure used in the parameterized form of particle mechanics and will be discussed in more detail below.) Should we wish to view the $v^{\rho}$ as coordinates (since they are now the independent variables), we would write the coordinate condition $v^{\mu}\left[g_{i j}, K^{i j}\right]=x^{\mu}$. The apparent singling out of a preferred coordinate frame does not imply a loss of general covariance. Any coordinate condition which can be satisfied generally, and in a unique way, can be re-expressed in covariant form. This can be seen most simply by starting with the example of the De Donder coordinate conditions. The conditions

$$
\mathfrak{g}^{\mu \nu}, \nu=0
$$

are equivalent to using as coordinates four scalar functionals of the metric, $\bar{v}^{\rho}$, defined as the four linearly independent solutions of

$$
\bar{v}_{; \mu}^{\rho} ; \mu \equiv\left(-{ }^{4} g\right)^{-\frac{1}{2}}\left(g^{\mu \beta} \bar{v}_{, \beta}^{\rho}\right)_{, \mu}=0 .
$$

Thus Eq. (2.4b) reduces to (2.4a) when one uses $\bar{v}^{\mu}$ as the coordinates $x^{\mu}$, but if one preferred to continue the discussion in explicitly covariant language, it would only be necessary to replace references to " $g_{\alpha \beta}$ in the preferred frame" by references to "the scalars $g_{\alpha \beta}$ defined by the equations

$$
\bar{g}_{\mu \nu}=g_{\alpha \beta}\left(\partial \bar{v}^{\alpha} / \partial \bar{x}^{\mu}\right)\left(\partial \bar{v}^{\beta} / \partial \bar{x}^{\nu}\right) \equiv g_{\alpha \beta} \bar{v}_{; \mu}^{\alpha} \bar{v}_{; \nu}^{\beta},
$$

where $\bar{g}_{\mu \nu}$ and $\bar{x}^{\mu}$ are the metric and coordinates of an arbitrary frame." For any other coordinate condition, those unique coordinate functions $v^{\rho}$ satisfying it are clearly scalar functions of the metric (by the assumed uniqueness), and statements like Eq. (2.5) can be used to emphasize the covariance of discussions employing coordinate conditions. In what follows, we use the language of coordinate conditions for simplicity, and write $x^{\mu}$ for $v^{\mu}$.

Returning now to the generator, we see that the $h_{\rho}(u, x) \delta x^{\rho}$ term is related only to space-time translations and would be the $T_{\rho}^{0} \delta x^{\rho}$ of the theory if the remaining part of the generator were found in the canonical form $u^{1} \delta u^{2}+u^{3} \delta u^{4}$ (to within a total variation). In fact, it can be shown (see Appendix $A$ ) that for any choice of gauge variables $v^{\rho}$ there exists an appropriate set of $u^{\rho}=\left(p_{1}, p_{2}, q_{1}, q_{2}\right)$ in terms of which $G$ has the canonical form

$$
G=\int d^{3} x\left[p_{a} \delta q_{a}+T^{0}{ }_{\mu}\left(p_{a}, q_{a}, x^{\rho}\right) \delta x^{\mu}\right]
$$

The significance of the result (2.6) is brought out by examining the parameter formalism of classical mechanics. In II, an example of this was given in terms of particle mechanics where one Lagrange multiplier occurred in the parameterized Lagrangian. In general relativity we have seen that there exist four Lagrange multipliers, $N$ and $\eta^{i}$. That this is characteristic of a field theory is brought out by the example of parameterized scalar meson theory. This case also provides an illustration of how the $u^{\rho}$, $v^{\rho}$, and $w^{\rho}$ variables enter in 
a simple theory and how one recovers the nonvanishing stress tensor $T^{0}{ }_{\mu}$ upon eliminating constraints and imposing coordinate conditions. In the first order action for the scalar meson theory

$$
I=\int d^{4} x\left[\pi \partial_{0} \phi-\frac{1}{2}\left(\pi^{2}+\phi^{2}-\phi_{k} \phi^{k}+2 \phi_{, k} \phi^{k}\right)\right],
$$

we introduce four parameters $\tau^{\mu}$ by writing $x^{\mu}=x^{\mu}(\tau)$. The action then becomes

$$
I=\int d^{4} \tau\left[\bar{\pi} \partial \phi / \partial \tau^{0}+\pi_{\mu} \partial x^{\mu} / \partial \tau^{0}+N^{\mu}\left(\pi_{\mu}-P_{\mu}\right)\right]
$$

where

$$
\begin{aligned}
P_{\mu}= & \frac{1}{2} \pi\left(\partial \phi / \partial \tau^{k}\right) \epsilon^{k 0 l m} \epsilon_{0 \mu j n} x^{j},{ }_{, 2} x^{n},{ }_{m} \\
& -\frac{1}{2} \phi^{l}\left(\partial \phi / \partial \tau^{k}\right) \epsilon^{k 0 m n} \epsilon_{l \mu \nu \sigma} x^{\nu},{ }_{m} x^{\sigma}{ }_{, n} \\
& \quad-\frac{1}{12}\left(\pi^{2}+\phi^{2}-\phi_{k} \phi^{k}\right) \epsilon^{0 m n n} \epsilon_{\mu \alpha \beta \gamma} x^{\alpha},{ }_{m} x^{\beta},{ }_{n} x^{\gamma},{ }_{p},
\end{aligned}
$$

and $\pi$ in Eq. (2.9) is to be expressed in terms of the variable $\bar{\pi}$ through

$$
\begin{aligned}
& \bar{\pi}=\frac{1}{3 !} \epsilon^{0 i j k} \epsilon_{l 0 m n} x^{l},{ }_{i} x^{m},{ }_{j} x^{n},{ }_{k} \pi \\
& \\
& \quad-\frac{1}{2} \phi^{l} \epsilon^{0 i j k} \epsilon_{l 0 m n} x^{0}, i x^{m}, j x^{n}, k .
\end{aligned}
$$

The $x^{\mu}{ }_{, \nu}$ mean $\partial x^{\mu} / \partial \tau^{\nu}$ and the $N^{\mu}(\tau)$ are four Lagrange multipliers introduced in order to call the coefficients of $\partial x^{\mu} / \partial \tau^{0}$ new momenta $\pi_{\mu}$. In this Lagrangian we regard $\bar{\pi}, \phi, \pi_{\mu}, x^{\mu}, \phi_{k}$ and $N^{\mu}$ as field variables in $\tau$ space. The resulting Lagrange equations break up into four constraint equations, ten equations involving first time derivatives and three algebraic constraint equations which will serve at the end to eliminate $\phi_{k}$ in terms of $\phi_{, k}$. The constraint equations (from varying $\left.N^{\mu}\right)$ allow us to choose $\pi_{\mu}$ as $w^{\rho}$ variables to be eliminated according to $\pi_{\mu}=P_{\mu}$. The ten remaining equations are equations for the $\tau^{0}$ derivatives of $\bar{\pi}, \phi, x^{\mu}$ and $\pi_{\mu}$. Thus the $\partial \pi_{\mu} / \partial \tau^{0}$ equations are the Bianchi identities while the $\partial x^{\mu} / \partial \tau^{0}$ equations serve to determine $N^{\mu}$. Since this leaves the $x^{\mu}$ undetermined we recognize them as a set of $v^{\rho}$ gauge variables leaving two dynamical $u^{\rho}$ variables, i.e., $\phi$ and $\bar{\pi}$.

The generator can easily be obtained to be

$$
G=\int d^{3} \tau\left(\bar{\pi} \delta \phi+\pi_{\mu} \delta x^{\mu}+T^{0}{ }_{\mu}{ }^{\delta} \tau^{\mu}\right) .
$$

The $T^{0}{ }_{\mu}{ }^{\prime}$ vanish as a consequence of the constraints and hence the generator has only $1+4$ terms. If we now impose coordinate conditions $x^{\mu}=\tau^{\mu}$, the generator reduces to standard canonical form

$$
G=\int d^{3} \tau\left(\bar{\pi} \delta \phi+P_{\mu} \delta \tau^{\mu}\right)
$$

$P_{\mu}$ now being the nonvanishing stress tensor components $T^{0}{ }_{\mu}$. Notice that in the special frame that we have chosen, $\bar{\pi}=\pi$ and $P_{\mu}$ is the $T^{0}{ }_{\mu}$ of conventional scalar meson theory as can be seen from Eqs. (2.10) and (2.9).

The situation in general relativity corresponds to being given the theory in already parameterized form analogous to Eq. (2.8) and the associated generator (2.11). One must "de-parameterize" the theory by imposing coordinate conditions and choosing $u^{\rho}$ variables such that the generator is left in canonical form. A second condition which must be simultaneously satisfied is that one must choose one's $u^{\rho}$ and $v^{\rho}$ variables in such a fashion that the Hamiltonian density, $-T^{0}$, does not depend explicitly on the coordinates, $\tau^{\mu}$. For example, in scalar meson theory, the coordinate conditions $x^{\mu}=(1+\phi) \tau^{\mu}$ would lead to $T^{0}{ }_{\mu}$ explicitly depending on the coordinates $\tau^{\mu}$, although it is still easy to recover canonical form by defining $\bar{\pi}+P_{\mu} \tau^{\mu}$ as the new momentum conjugate to $\phi$. While in scalar meson theory it is obviously incorrect to mix $\phi$ and $x^{\mu}$ by choosing $v^{\mu}=x^{\mu} /(1+\phi)$ as the gauge variables, in general relativity we have no a priori criteria to determine when a particular choice of gauge variable has involved some canonical parts.

\section{REDUCTION TO CANONICAL FORM}

The generator arising from the Lagrangian (2.2) has the form

$$
G=\int d^{3} x\left({ }^{3} g\right)^{\frac{1}{2}}\left(-g^{i j} \delta K_{i j}-\delta K+T_{\mu}^{0} \delta x^{\mu}\right) .
$$

As seen from Eq. (2.6) the achievement of canonical form requires that $G$ be written as a sum of six terms. To this end let us introduce the variables

$$
\pi^{i j}=-\left({ }^{3} g\right)^{\frac{1}{2}}\left(K^{i j}-g^{i j} K\right) .
$$

The generator then becomes

$$
G=-\int d^{3} x g_{i j} \delta \pi^{i j}
$$

where we had dropped the $T^{0}{ }_{\mu}{ }^{\prime}$ terms since they vanish by the constraint equations. While the generator is now a sum of six terms, the dynamic and gauge character of the variables has not yet been established. Because of the bilinear form of the generator in (3.3), an orthogonal decomposition of the tensors $g_{i j}$ and $\pi^{i j}$ into transverse and longitudinal parts maintains this simple form while allowing the $u^{\rho}, v^{\rho}, w^{\rho}$ character of the variables to be recognized. For a general symmetric array $f_{i j}=f_{j i}$ we write

where

$$
f_{i j}=f_{i j}{ }^{T T}+f_{i j}{ }^{T}+f_{i, j}+f_{j, i},
$$

$$
\begin{aligned}
f_{i j}{ }^{T} & =\frac{1}{2}\left[\delta_{i j} f^{T}-\left(1 / \nabla^{2}\right) f^{T}, i j\right], \\
f_{i j}{ }^{T}, j & =0, \quad f_{i i}{ }^{T}=f^{T},
\end{aligned}
$$

and

$$
f_{i j}{ }^{T T}, j=0, \quad f_{i i}{ }^{T T}=0 .
$$


Thus $f_{i j}{ }^{T T}$ is the transverse traceless part of $f_{i j}$ and so has only two independent components while $f^{T}$ is the trace of the transverse part. The remainder, $f_{i, j}+f_{j, i}$, contains the longitudinal parts of $f_{i j}$. Each of the six independent quantities $f_{i j}{ }^{T T}, f^{T}$, and $f_{i}$ can be uniquely obtained as a linear functional of $f_{i j}$. For instance,

$$
\begin{aligned}
f^{T} & =f_{i i}-\left(1 / \nabla^{2}\right) f_{i j, i j}, \\
f_{i} & =\left(1 / \nabla^{2}\right)\left[f_{i j, j}-\frac{1}{2}\left(1 / \nabla^{2}\right) f_{k j, k j i}\right] .
\end{aligned}
$$

Note that this decomposition is coordinate dependent and involves only ordinary derivatives. Thus $1 / \nabla^{2}$ is the inverse of the flat space Laplacian operator, and the decomposition ignores the position of tensor indices. This use of ordinary derivatives permits one to commute the decomposition and the variation in the generator, e.g., $\delta\left(\pi^{i j T}\right)=\left(\delta \pi^{i j}\right)^{T}$.

Upon applying this decomposition to $g_{i j}$ and $\pi^{i j}$, the generator becomes

$$
\begin{array}{r}
G=\int d^{3} x\left\{\delta g_{i j}{ }^{T T} \pi^{i j T T}+g^{T}, i i \delta\left[-\frac{1}{2}\left(1 / \nabla^{2}\right) \pi^{T}\right]\right. \\
\left.-2 \pi^{i j},{ }_{j} \delta g_{i}\right\} .
\end{array}
$$

In obtaining this form, we have freely integrated by parts and discarded surface terms as well as total variations. Note that the generator of Eq. (3.8) is still a sum of six terms. This result could not be obtained by applying our decomposition to $\mathfrak{g}^{i j}$ and $K_{i j}$ in Eq. (3.1) as that generator is not bilinear in these variables.

The constraint equations in terms of these variables read:

$$
\begin{aligned}
{ }^{3} g{ }^{3} R+\frac{1}{2} \pi^{2}-\pi^{i j} \pi_{i j} & =0, \\
\pi^{i j}{ }_{\mid j} & =0 .
\end{aligned}
$$

When these are expressed according to the above decomposition, one sees that the leading term, i.e., the only linear term, in Eq. (3.9a) is $g^{T}{ }_{, i i}$ and the leading term of (3.9b) is $\pi^{i},{ }_{j j}+\pi^{j}, i j=\pi^{i j},{ }_{j}$. By a perturbation argument as given in II, one verifies that the constraint equations may be solved for these four quantities and hence we may choose them as our $w^{\rho}$ variables. That this is advantageous follows from the fact that these quantities appear directly in the generator (3.8).

Turning next to the identification of the remaining eight variables, one observes that $g_{i j}{ }^{T T}$ and $\pi^{i j T T}$ are the dynamical $u^{\rho}$ variables of linearized theory, ${ }^{1}$ while $g_{i}$ and $-\frac{1}{2}\left(1 / \nabla^{2}\right) \pi^{T}$ are the $v^{\rho}$ there. Again following the perturbation interation scheme of II, we see this characterization maintained in the full theory, i.e., the $\partial_{0}\left[-\frac{1}{2}\left(1 / \nabla^{2}\right) \pi^{T}\right]$ and $\partial_{0}\left(g_{i}\right)$ equations serve to determine $N$ and $\eta^{i}$, respectively, in a perturbation series. In accordance with the discussion of Sec. II, we may regard these quantities as the $v^{\mu}$ which serve as the basic independent variables by imposing the coordinate conditions

$$
\begin{aligned}
-\frac{1}{2}\left(1 / \nabla^{2}\right) \pi^{T} & =x^{0}, \\
g_{i} & =x^{i} .
\end{aligned}
$$

These coordinate conditions are discussed in more detail below. Our generator now reads

$$
G=\int d^{3} x\left(\pi^{i j T T} \delta g_{i j}{ }^{T T}+g^{T},{ }_{i i} \delta x^{0}-2 \pi^{i j},{ }_{j} \delta x^{i}\right),
$$

where $g^{T}, i i$ and $\pi^{i j}, j$ are to be viewed as functions of $g_{i j}{ }^{T T}$ and $\pi^{i j T T}$ and possibly $v^{\mu}=x^{\mu}$ according to the solutions of Eqs. (3.9).

The generator is now clearly in canonical form with $\pi^{i j T T}$ the momenta canonically conjugate to $g_{i j}{ }^{T T}$. Similarly we have that the Hamiltonian density, $\mathfrak{H}$, is

$$
\mathfrak{H}=-T^{0}{ }_{0}=-g^{T},{ }_{i i}\left[g_{i j}{ }^{T T}, \pi^{i j T T}, x^{\mu}\right],
$$

and the momentum density is

$$
T_{i}^{0}=-2\left(\pi^{i},{ }_{j j}+\pi^{j}, i j\right)=-2 \pi^{i j}{ }_{, j},
$$

since the generator is in the form of Eq. (2.6). It should perhaps be emphasized that the quantities $g^{T}{ }_{, i i}$ and $\pi^{i j}{ }_{j}$, appearing in Eqs. (3.11), (3.12) and (3.13), are abbreviations for their values in terms of $g_{i j} T T, \pi^{i j T T}$ and $x^{\mu}$ as obtained from solving the constraint equations (3.9). Thus the solutions of Eqs. (3.9) for $g^{T}, i i$, etc., in terms of the canonical variables are not in general divergences, i.e., the Hamiltonian and momentum densities are not divergences, just as they are not in other physical systems.

From the definition of the generator, one may now read off the Poisson brackets. The fundamental [ordinary] Poisson brackets for the two independent canonical pairs of variables are

$$
\begin{aligned}
& {\left[g_{i j}{ }^{T T}(\mathbf{r}, t), \pi^{m n T T}\left(\mathbf{r}^{\prime}, t\right)\right]=\delta^{m n}{ }_{i j}\left(\mathbf{r}-\mathbf{r}^{\prime}\right),} \\
& {\left[g_{i j}{ }^{T T}(\mathbf{r}, t), g_{m n}{ }^{T T}\left(\mathbf{r}^{\prime}, t\right)\right]=0,} \\
& {\left[\pi^{i j T T}(\mathbf{r}, t), \pi^{m n T T}\left(\mathbf{r}^{\prime}, t\right)\right]=0,}
\end{aligned}
$$

where $\delta^{m n}{ }_{i j}\left(\mathbf{r}-\mathbf{r}^{\prime}\right)$ is the transverse traceless $\delta$ function defined as in linearized theory by

$$
\begin{gathered}
\delta^{m n_{i j}}=\delta^{n m}{ }_{i j}=\delta^{m n}{ }_{j i}=\delta^{i j}{ }_{m n}, \\
\delta^{m m_{i j}}=\delta^{m n}{ }_{i i}=0, \\
\frac{\partial}{\partial x^{i}} \delta_{i j}^{m n}\left(\mathbf{r}-\mathbf{r}^{\prime}\right)=0 .
\end{gathered}
$$

The functions $\delta^{m n}{ }_{i j}(\mathbf{r})$ are thus independent of the metric.

In terms of the $\pi^{i j}$ variables, the action integral becomes

$$
\begin{array}{r}
I=\int d^{4} x\left[-g_{i j} \partial_{0} \pi^{i j}+N\left({ }^{3} g\right)^{-\frac{1}{2}}\left({ }^{3} g{ }^{3} R+\frac{1}{2} \pi^{2}-\pi_{i j} \pi^{i j}\right)\right. \\
\left.+2 \eta_{i} \pi^{i j}{ }_{\mid j}-2\left({ }^{3} g\right)^{\frac{1}{2}}\left(N_{\mid i}-\eta^{j} K_{i j}\right)^{\mid i}\right] .
\end{array}
$$


Inserting the differential constraints and the coordinate conditions (3.10), the action reduces, to within divergences, to

$$
I=\int d^{4} x\left\{\pi^{i j T T} \partial_{0} g_{i j}{ }^{T T}-\mathfrak{F} C\left[g_{i j}{ }^{T T}, \pi^{i j T T}, x^{\mu}\right]\right\} .
$$

Equation (3.17) represents the action for a system of two unconstrainted degrees of freedom and no longer makes reference to the originally redundant number of variables. The Lagrange equations of motion arising from varying (3.17) are clearly consistent with the Poisson bracket equations of motion arising from Eqs. (3.14) with $3 C$ playing the role of the Hamiltonian density. This verifies the internal consistency of the Poisson bracket relations (3.14) for the theory. ${ }^{1 a}$ This result was of course guaranteed since the Lagrangian of Eq. (3.17) is in the standard $p \dot{q}-H(p, q)$ form.

The coordinate conditions of Eq. (3.10) can be restated in a form in which the coordinates $x^{\mu}$ do not appear explicitly:

$$
\begin{gathered}
\pi^{T}=0, \\
\frac{1}{2}\left(g_{i, j}+g_{j, i}\right)=\delta_{i j} .
\end{gathered}
$$

Alternately, one may re-express Eqs. (3.18) in terms of conditions on the $g_{i j}$ and $\pi^{i j}$. Thus we have

$$
\begin{aligned}
\nabla^{2} \pi^{T} & \equiv \pi^{i i},{ }_{, j}-\pi^{i j}, i j=0, \\
\nabla^{2} g_{i} & \equiv g_{i j, j}=0 .
\end{aligned}
$$

These differential conditions involve Laplacian operators acting on Eqs. (3.10). They are equivalent to Eqs. (3.10) only when one retains the appropriate boundary conditions. To recover Eq. (3.18) from Eq. (3.19) one must invoke the condition that the metric be asymptotically flat at spacial infinity. Similarly Eq. (3.10a) can be recovered from Eq. (3.18a) by the same requirement. Thus the equation giving the time derivative of $\pi^{T}$ in terms of $N$ reduces asymptotically to

$$
\partial_{0}\left[-\frac{1}{2}\left(1 / \nabla^{2}\right) \pi^{T}\right]=N=1 \text {, }
$$

since $N=\left(-g^{00}\right)^{-\frac{1}{2}}=1$ for flat space. Similarly, the equation for $\eta_{i}$ reduces, at spacial infinity, to

$$
\eta_{i, j}+\eta_{j, i}=\partial_{0}\left(g_{i, j}+g_{j, i}\right)=0,
$$

${ }^{1 \mathrm{a}}$ Note added in proof.- - The primary consistency check is that the equations of motion obtained from Eq. (3.17) be equivalent to the Einstein equations. It is indeed possible to show this by inserting the constraints and coordinate conditions into the Einstein equations [obtained by varying Eq. (3.16)] and comparing with those from Eq. (3.17). This shows that the Hamiltonian, $\int d^{3} x \mathcal{F}$, consistently generates, for general relativity, translations in the chosen time coordinate. Similarly, the canonical momentum density [which arises from Eq. (3.17)], $T^{C 0} i_{i}=-\pi^{k j T T} g_{k j}{ }^{T T}, i$ generates space translations. By first showing that $T^{C 0_{i}}$ differs from $-2\left(g_{i m} \pi^{m j}\right), j \equiv-2 \pi_{i}^{i}, j\left[G^{T T}, \pi^{T T}\right]$ by a divergence of the canonical variables, one then sees that $T_{i}^{0}=-2 \pi^{i j}{ }_{i}$ obtained from the generator (3.11) differs from $T^{C{ }^{C}}{ }_{i}$ only by a divergence. Thus $T^{0}{ }_{i}$ consistently generates translations in $x^{i}$.

Also, it might be noted that the numerical value of the standard Einstein-Tolman and Landau-Lifshitz surface integral forms for the energy and momentum agree with ours, as derived by the canonical formalism. and hence $\eta_{i}$ is a constant vector which for simplicity we choose to be zero. With this choice, our coordinates $x^{\mu}$ are rectangular at infinity.

The coordinate conditions we use are given in a form analogous to Eq. (2.4a), not Eq. (2.4b). Consequently, the equality $v^{i}=g_{i}$ holds only in the preferred frame. A deeper investigation of the existence and uniqueness of coordinates satisfying Eqs. (3.19), leading to a construction of these $v^{\mu}$ from the $g_{\mu \nu}$ of an arbitrary frame, would be desirable though perhaps quite difficult. However, it is easy to see that these coordinate conditions are consistent with Einstein's equations in the sense that, given initial values of $g_{i j}$ and $\pi^{i j}$ at time $t=0$ compatible with Eqs. (3.19), a unique solution for $g_{i j}$ and $\pi^{i j}$ at later times is obtained by integrating the Einstein equations. The proof would employ a perturbation argument, as in the discussion of the constraint equations in II.

The canonical formalism obtained above is very similar to that of linearized theory as discussed in I, with of course a more complicated expression for the Hamiltonian. Thus $\pi^{i j T T}$ linearizes to $\Gamma^{0}{ }_{i j}{ }^{T T}$ while $\pi^{T}$ linearizes to the purely longitudinal part of $\Gamma^{0}{ }_{i j}$, i.e., $\Gamma^{0}{ }_{i j}{ }^{L}$. The "radiation gauge" in I was defined precisely by the transversality condition on $g_{i j}$, i.e., Eq. (3.19b) and the vanishing of $\Gamma_{i j}^{0}{ }_{i j}$. Similarly, as was discussed in II, a solution of Eq. (3.9a) for $g^{T}, i i$ to quadratic order yields the linearized Hamiltonian. However, if in the full theory one simply imposed the coordinate conditions $g_{i j, j}=0$ and $\Gamma_{i j}^{0}{ }_{i j}=0$, one would not obtain a canonical form for the generator. Thus the introduction of the $\pi^{i j}$ variables, with the consequent reduction of the generator to a sum of six terms, was essential to obtain a canonical form. It is easy to exhibit other forms of the generator which also only involve six terms. For example, an infinite class of these is

$$
G=-\int d^{3} x \bar{g}^{i j} \delta \bar{K}_{i j}
$$

where

$$
\begin{aligned}
\bar{g}^{i j} & =\left({ }^{3} g\right)^{\lambda} \mathfrak{g}^{i j}, \\
K_{i j} & =\left({ }^{3} g\right)^{\lambda}\left[\bar{K}_{i j}-\frac{1}{2}\left(\lambda+\frac{3}{2}\right) g_{i j} g^{m n} \bar{K}_{m n}\right]
\end{aligned}
$$

for any $\lambda$. Further, the class of generators formed from that of (3.22) by raising and lowering indices, i.e.,

$$
G=\int d^{3} x \bar{g}_{i j} \delta \bar{K}^{i j}
$$

differs from (3.22) only by a total variation. The generator we have discussed corresponds to the case $\lambda=-\frac{1}{2}$. If one were to make the orthogonal decomposition of Eq. (3.4) on these new generators and impose coordinate conditions analogous to Eqs. (3.10), one would again obtain a canonical form. The new canonical variables $\bar{g}_{i j}{ }^{T T}$ and $\bar{K}^{i j T T}$ are related to the ones discussed here by a canonical transformation which involves a coordinate transformation. 


\section{CONSERVATION LAWS}

In the preceding section we have seen that the first half of the problem stated in Sec. II, namely the reduction of the generator to canonical form, has been solved. We shall see in this section (and in more detail in Appendix B) that the second half of the problem, namely the coordinate independence of the Hamiltonian and momentum densities, has in fact, also been solved. Since functionals of $g_{i j}$ and $\pi^{i j}$ have been used as coordinates, one would expect a priori that they appear in the expressions for $T_{\mu}^{0}$. In broad outline, the reason why this is avoided with our coordinate conditions is that the coordinates themselves do not appear explicitly in $g_{i j}$ and $\pi^{i j}$. Thus while $g_{i}=x^{i}$, only $\frac{1}{2}\left(g_{i, j}+g_{j, i}\right)=\delta_{i j}$ appears in $g_{i j}$. Similarly, only $\pi^{T}=-2 \nabla^{2} x^{0}=0$ appears in the decomposition of $\pi^{i j}$. In fact, as the constraint equations (3.9) contain only $g_{i j}$ and $\pi^{i j}$ and no explicit $x^{\mu}$, it becomes difficult at first sight to imagine how an explicit $x^{\mu}$ dependence can appear in their solutions. ${ }^{2}$ However, the process of solving the differential equations introduces operators such as $1 / \nabla^{2}$, which if applied to $\pi^{i i}=\pi^{T}+2 \pi^{i}{ }_{i}$ leads to a term $\left(1 / \nabla^{2}\right) \pi^{T}$ $=-2 x^{0}$. Similarly, $\left(1 / \nabla^{2}\right) g_{i j, j}$ would lead to the term $x^{i}$. As is shown in the explicit proof in Appendix B, however, terms of this sort never appear and the structures that do actually arise are coordinate independent.

We may now use this coordinate independence of $T_{0}^{0}$ to establish a set of conservation laws. Since, in Eq. (3.17), $\mathfrak{F}$ does not depend explicitly on $x^{\mu}$, the standard techniques of Lagrangian field theory allow one to derive a stress tensor $\bar{T}^{\mu}{ }_{\nu}$ satisfying

$$
\bar{T}_{\nu, \mu}^{\mu}=0 \text {. }
$$

We have given an expression in this paper for $\bar{T}_{0}^{0}=$ $T_{0}^{0}$. The Lagrangian of Eq. (3.17) is furthermore manifestly covariant under three dimensional rigid rotations. This implies that $\bar{T}^{i j} \equiv \bar{T}^{i}{ }_{j}$ is a symmetric three-tensor and hence a conservation law for three dimensional angular momentum holds. Thus if we define

$$
M^{i \mu j}=x^{i} \bar{T}^{\mu i}-x^{j} \bar{T}^{\mu i},
$$

where $\bar{T}^{0 j} \equiv \bar{T}_{j}^{0}$ then one has

$$
M^{i \mu i}{ }_{, \mu}=0 .
$$

The above results were established without recourse to Lorentz covariance. The latter is needed to establish the remaining conservation law for $M^{i \mu 0}$. We hope to discuss later the Lorentz transformation properties of the canonical formalism.

\section{DISCUSSION}

In the previous sections, general relativity has been cast into a canonical form. Explicit canonical variables

\footnotetext{
2 The apparent appearance of explicit $x^{i}$ dependence in the integral representation of $\left(1 / \nabla^{2}\right)\left[\right.$ i.e., in $\left.(4 \pi)^{-1} \int d^{3} x^{\prime}\left(1 /\left|\mathbf{x}-\mathbf{x}^{\prime}\right|\right)\right]$ is spurious since the $\nabla^{2}$ operator is invariant under rotations and translations.
}

have been obtained. Since these are grouped as two pairs of unconstrained conjugate variables, the fundamental Poisson bracket relations follow just as in ordinary field theories. The Hamiltonian density was shown to have no explicit dependence on the coordinates. The energy of the field is defined as the numerical value of the Hamiltonian for any solution of the equations of motion. Thus one has

$$
E=-\int d^{3} x g^{T}, i i=-\int g^{T},{ }_{i} d S_{i},
$$

where $d S_{1}=d x^{2} d x^{3}$, etc., are the rectangular elements of a surface at spacial infinity. The transition from a volume integral to a surface integral cannot of course be made for the Hamiltonian where $g^{T}{ }_{, i i}$ is expressed in terms of canonical variables [according to the solutions of the constraint equations (3.9)] and hence is not then a divergence. The energy, however, may be directly evaluated from the knowledge of $g^{T}$ on the surface. A similar expression holds for the spacial components of the momentum four-vector. A fuller discussion of these formulas for momentum and energy will be given in a following paper, ${ }^{3}$ where the results obtained here are extended to include a matter system coupled to the gravitational field. The criteria for the existence of gravitational radiation will also be given there.

One of the principal purposes of this classical investigation is, of course, to serve as a basis for a quantum theory of relativity. One might suppose that a quantum theory could now be obtained by merely replacing the Poisson bracket relations of Eq. (3.14) by the corresponding commutators. One could do this, presumably, by choosing some Hermitian ordering for the Hamiltonian, though this is highly non-unique. Indeed, one would obtain in this manner a quantum theory for the two canonical degrees of freedom. However, these two degrees of freedom do not represent the full statement of general relativity: one also has equations to determine $g_{0 \mu}$, for example, which are not part of this canonical theory as such. Furthermore, one encounters these variables as soon as one makes a Lorentz transformation from the initial frame, ${ }^{4}$ an operation that must be allowed for any sensible quantum theory. Since the equations defining $g_{0 \mu}$ are now quantum ones, one must establish the consistency of the ordering of the Hamiltonian and the $g_{0 \mu}$ equations in one frame with those in another frame. Finally, classically we saw, that there was an infinite number of a priori equivalent sets of simple canonical variables [Eq. (3.22)] with conserved Hamiltonians, each of

${ }^{3}$ R. S. Arnowitt, S. Deser, and C. W. Misner, Phys. Rev. 118, (1960) (to be published).

${ }^{4}$ In electrodynamics, one similarly has that the gauge functions (i.e., the scalar potential and longitudinal part of the vector potential) mix with the dynamical variables (i.e., the transverse part of the vector potential) when a Lorentz transformation is made. 
which could of course be used as a basis for such a quantum scheme; quantum mechanically, however, the relation between these sets of variables need no longer be one of a unitary transformation, due to the operator character of the variables. Hence these are no longer equivalent starting points for quantization. Further, the classical canonical transformations among these sets may well make the coordinates associated with one set functions of both the canonical variables and coordinates of the other. This would lead to the phenomenon in the quantum theory of the coordinates of one set being $q$-numbers when expressed in terms of the variables of the other set, as was discussed in II.

In view of the many ambiguities which could arise in an attempt to quantize consistently at this level, it would seem more fruitful to return to the action in four dimensional form, i.e., $\mathrm{g}^{\mu \nu} R_{\mu \nu}\left(\Gamma^{\alpha}{ }_{\lambda \rho}\right)$ and try to repeat our reduction to canonical form within the framework of quantum theory. There, one can use the manifest Lorentz covariance of the original Lagrangian as an aid in proving the Lorentz covariance of the canonical quantum form that should arise. Further, the ordering ambiguities are drastically reduced. Since at most cubic terms enter in this Lagrangian, one can show easily that there is at most a simple three parameter family of available quantum Lagrangians, all of which are generally covariant. These different orderings of the Lagrangian differ from each other only by double commutators, i.e., effects of order $\hbar^{2}$. The basic requirement of consistency between the Lagrange and Heisenberg equations of motion should single out one of these forms since the double commutators do not contribute to the Lagrange equations but presumably affect the Heisenberg ones. In an investigation based on the four dimensional form of the quantum Lagrangian one ultimately expects to arrive at canonical forms very similar to those obtained here. Thus the classical results represent an excellent guide in formulating the quantum theory.

\section{APPENDIX A}

We show here that for any choice of gauge variables, $v^{\rho}$, the canonical form (2.6) can be reached from the generator

$$
G=\int d^{3} x\left[f_{\rho}(u, x) \delta u^{\rho}+h_{\rho}(u, x) \delta x^{\rho}\right]
$$

where we have chosen coordinates $v^{p}=x^{p}$. The first four terms, $f_{\rho} \delta u^{\rho}$, can always be reduced to a sum of two terms by a classical theorem in differential forms. ${ }^{5}$ In applying this theorem, it is necessary to view $x^{\mu}$ as parameters, not to be varied. Thus we have

$$
f_{\rho} \delta u^{\rho}=p_{a} \delta_{u} q_{a}+\delta_{u} W(u, x)
$$

${ }^{5}$ See, for example, J. M. Thomas, Differential Systems, Am. Math. Soc. Colloq. Pub. XXI, 1937, and the appendix in II. where $a=1,2$ and $p_{a}, q_{a}$ are functions of $u^{\rho}$ and $x^{\rho}$. To reinstate total variations, therefore, we write

$$
\begin{aligned}
\delta_{u} & =\delta-\delta_{x} \\
& =\delta-\delta x^{\mu} \partial / \partial x^{\mu}
\end{aligned}
$$

and obtain

$$
\begin{aligned}
G=\int d^{3} x\left[p_{a} \delta q_{a}+\left\{h_{\mu}-p_{a}\left(\partial q_{a} / \partial x^{\mu}\right)\right.\right. & \\
& \left.\left.-\partial W / \partial x^{\mu}\right\} \delta x^{\mu}\right]
\end{aligned}
$$

where the total variation $\delta W$ has been discarded in $G$. When the $u^{\rho}$ have been eliminated in terms of $p_{a}, q_{a}$, and $x^{\mu}$ in the coefficient of $\delta x^{\mu}$ of Eq. (A.4), one has reached the desired result, Eq. (2.6). However, the $T^{0}{ }_{\mu}$ so obtained (i.e., the coefficient of $\delta x^{\mu}$ ) will in general depend explicitly on $x^{\mu}$.

\section{APPENDIX B}

We demonstrate here the independence of the Hamiltonian and momentum densities on the coordinates we have chosen. These functions arise as solutions of the constraint equations (3.9). We will make use of a perturbation interation solution in our proof similar to the one discussed in II.

The care that is required in the analysis is illustrated by the apparent contradiction between the equations $-\frac{1}{2}\left(1 / \nabla^{2}\right) \pi^{T}=x^{0}$ and $\pi^{T}=-2 \nabla^{2} x^{0}=0$. This would lead to difficulties in the generator where the term $g^{T} \delta \pi^{T}$ arises, which we have rewritten as $g^{T},{ }_{i i} \delta\left[\left(1 / \nabla^{2}\right) \pi^{T}\right]$. (As we shall see below, setting $\pi^{T}$ to zero in the constraint equations leads to correct results since effectively no structures such as $\left(1 / \nabla^{2}\right) \pi^{T}$ appear in their solutions.) To give these operations meaning we shall therefore introduce a convergence factor such as $\exp (-\alpha r)$ to multiply the $x^{\mu}$ in Eqs. (3.10) :

$$
\begin{aligned}
-\frac{1}{2}\left(1 / \nabla^{2}\right) \pi^{T} & =x^{0} \exp (-\alpha r), \\
g_{i} & =x^{i} \exp (-\alpha r) .
\end{aligned}
$$

The limit $\alpha \rightarrow 0$ will of course be taken at the end of the analysis. This means that neither $\pi^{T}$ nor $g_{i, j}+g_{j, i}$ are independent of $x^{\mu}$ and thus $g_{i j}$ and $\pi^{i j}$ regain their explicit coordinate independence only in the limit $\alpha \rightarrow 0$. We will use in the discussion the formulas

$$
\lim _{\alpha \rightarrow 0}\left(1 / \nabla^{2}\right)\left[\pi^{T} f(\mathbf{r})\right]=0,
$$

if $f(\mathbf{r})$ approaches zero at infinity as $r^{-n}, n>0$, while

$$
\lim _{\alpha \rightarrow 0}\left(1 / \nabla^{2}\right)\left[g_{i, j} f(\mathbf{r})\right]=0,
$$

if $f(\mathbf{r})$ approaches zero as $r^{-n}, n>2$. Similar theorems hold when higher derivatives act on $g_{i}$ or $\pi^{T}$ and when products of these functions occur. The $f(\mathbf{r})$ that will arise, actually go to zero much faster than the minimal limits stated in the above theorems. 
We begin by examining the $\pi^{i j}$ constraint equation (3.9b) which we rewrite as an equation for $\rho^{i} \equiv \pi^{i}, j j$ $+\pi^{i}, i j=\pi^{i j}, j$ :

$$
\begin{aligned}
\rho^{i}=-\left(\pi^{l m T T} \Gamma^{i}{ }_{l m}+\pi^{l m T} \Gamma^{i}{ }_{l m}\right)-\left\{\left(1 / \nabla^{2}\right)\right. \\
\left.\times\left[\rho^{l}{ }_{, m}+\rho^{m}, l-\left(1 / \nabla^{2}\right) \rho^{p}, p m l\right]\right\} \Gamma^{i}{ }_{l m} .
\end{aligned}
$$

We view this as an equation to be solved by iteration on $\rho^{i}$. Although these equations must eventually be solved simultaneously with Eq. (3.9a), it is first convenient to solve Eqs. (B.3) for $\rho^{i}$ in terms of $\Gamma^{l}{ }_{i j}$, $\pi^{l m T T}, \pi^{l m T}$. The zeroth step of the iteration, which is just the first bracket on the right-hand side of Eqs. (B.3), contains the linear theory's expression for $T^{0}{ }_{i}$ when one remembers that $\pi^{T}=0$ in the limit $\alpha=0$. The parts of the first iteration involving $\pi^{T}$ have the form

$$
\left[\left(1 / \nabla^{2}\right)\left(\pi^{T} \Gamma^{l}{ }_{s s, m}\right)\right] \Gamma^{i}{ }_{m l} \text {. }
$$

The coefficient $\Gamma_{s s, m}^{l}$ of $\pi^{T}$ contains structures depending on $g_{i j}{ }^{T T}, g^{T}$, and $g_{i, j}$. Both $g_{i j}{ }^{T T}$ and $g^{T}{ }_{, i}$ must vanish at infinity since $g_{i j}$ approaches $\delta_{i j}$ there. The terms containing any $g_{i j}{ }^{T T}$ or $g^{T}$ factor give no contribution according to Eq. (B.2a). The remaining terms contain only $\pi^{T}$ and $g_{i, j}$ and can be seen to vanish by direct insertion of Eq. (B.1). In higher iterations, $\pi^{T}$ always appears in factors identical to those treated in the first iteration, e.g., $\left(1 / \nabla^{2}\right)\left(\pi^{T} \Gamma_{s n, m}^{l}\right)$. Hence the argument goes through for all higher orders and $x^{0}$ does not appear explicitly in the solution $\rho^{i}=\rho^{i}\left[\pi^{i j T T}, \Gamma^{i}{ }_{m l}\right]$. Further, the only places where $x^{i}$ might appear in the solution for $\rho^{i}$ are in the $g^{T}$ arising in $\Gamma_{m l}^{i}$ and from the $\Gamma^{i}{ }_{m l}$ in terms of the type $\left(1 / \nabla^{2}\right)\left(\pi^{m l T T} \Gamma^{i}{ }_{m l}\right)$. On account of theorems of the sort given in Eqs. (B.2) the latter type of terms are actually independent of $x^{i}$.

Turning next to Eq. (3.9a) we will investigate its solution for $g^{T}$ in terms of $g_{i j}{ }^{T T}$ and $\pi^{i j}$. We rewrite here the orthogonal decomposition (Eq. 3.4) of $g_{i j}$ as

where

$$
g_{i j}=\delta_{i j}+g_{i j}{ }^{T T}+g_{i j}{ }^{T}+h_{i, j}+h_{j, i},
$$

$$
h_{i}=g_{i}-x^{i}=x^{i}[\exp (-\alpha r)-1] .
$$

The purpose of this rearrangement is to extract the leading term $-g^{T}, i i$ in ${ }^{3} R$. Eq. (3.9a) then takes the form

$$
\begin{aligned}
\rho \equiv-g^{T},{ }_{, i i} & =\mathcal{H C}_{2}\left(g_{i j}{ }^{T T}, h_{i}, \pi^{i j}\right)+\mathcal{H C}_{3}\left(g_{i j}{ }^{T T}, h_{i}, \pi^{i j}\right) \\
& +f_{2}\left(g^{T}, g_{i j}{ }^{T T}, h_{i}, \pi^{i j}\right)+f_{3}\left(g^{T}, g_{i j}{ }^{T T}, h_{i}, \pi^{i j}\right) .
\end{aligned}
$$

Here $\mathfrak{H C}_{2}$ contains all quadratic terms independent of $\rho$ and $\mathfrak{F C}_{3}$ the cubic and higher terms independent of $\rho ; f_{2}$ contains the other quadratic terms while $f_{3}$ contains the other higher order terms. The zero'th iteration on $\rho$ is, of course, just $\mathfrak{F C}_{2}+\mathfrak{F C}_{3}$. This contains as the leading term in the limit $\alpha=0$ the linear theory's Hamiltonian density. The next iteration then proceeds by substitution of $-\left(1 / \nabla^{2}\right)\left(\mathcal{F C}_{2}+\mathcal{H C}_{3}\right)$ for $g^{T}$ in $f_{2}$ and $f_{3}$. We first discuss the $\left(1 / \nabla^{2}\right) \mathfrak{H C}_{2}$ term. Those parts of $\mathcal{F C}_{2}$ depending on $g_{i j}{ }^{T T}$ or $\pi^{i j T T}$ bring in no $x^{\mu}$ dependence by virtue of Eq. (B.2b). We have used here the fact that the canonical variables vanish very rapidly at infinity for a system of finite energy. ${ }^{6}$ The terms in $\left(1 / \nabla^{2}\right) \mathcal{H C}_{2}$ independent of canonical variables yield a constant, $C$, in the limit $\alpha=0$ [using Eq. (B.1)] by direct calculation with a Coulomb Green's function for $1 / \nabla^{2}$. However, the boundary condition that $g^{T}$ vanish at infinity ${ }^{7}$ means that one must add to the Coulomb integral solution a constant that cancels $C$. In $\left(1 / \nabla^{2}\right) \mathfrak{H C}_{3}$ the cubic and higher terms independent of canonical variables vanish by direct calculation in the limit $\alpha=0$, while by theorems of the type quoted in Eqs. (B.2) the remaining terms are independent of $x^{\mu}$. Higher order iterations give rise to structures of the form $\left(1 / \nabla^{2}\right)\left(f_{2}+f_{3}\right)$ where in $f_{2}$ and $f_{3}, g^{T}$ has been replaced by its lower order value. That these possess no explicit $x^{\mu}$ dependence follows in a fashion analogous to the treatment of $\left(1 / \nabla^{2}\right) \mathcal{H}_{3}$.

The above discussion has established that the two series

$$
\begin{aligned}
\rho^{i} & =\rho^{i}\left[\pi^{i j T T}, g_{i j}{ }^{T T}, \rho\right], \\
\rho & =\rho\left[g_{i j}{ }^{T T}, \pi^{i j T T}, \rho^{i}\right]
\end{aligned}
$$

contain no explicit dependence on $x^{\mu}$ in the limit $\alpha=0$. Also the total solution, obtained by cross iteration between the $\rho^{i}$ and $\rho$ equations, is found in dependent of $x^{\mu}$, establishing finally the fact that $T^{0}{ }_{\mu}$ has no explicit $x^{\mu}$ dependence.

The result that we have obtained implies that $\mathfrak{H C}$ and $T_{i}^{0}$ arise unambiguously from the constraint equations (3.9) by simply inserting there the coordinate conditions

$$
\pi^{T}=0, \quad g_{i j, j}=0
$$

without regard to the possible difficulties discussed at the beginning of this appendix.

\footnotetext{
${ }^{6}$ For example, if we have a packet of radiation confined to a finite domain one could assume that the canonical variables went to zero exponentially at infinity with no loss of generality as the canonical variables are then rigorously zero there.

${ }^{7}$ In the orthogonal breakup (3.4) for $g_{i j}$, the boundary condition $g^{T}=-2$ at infinity was employed so that, with $g_{i}=x^{i}$, the metric approach $\delta_{i j}$. In the form (B.5), $g^{T}$ has been redefined (for convenience) to approach zero at infinity to comply with the same boundary condition. This does not, of course, affect any results.
} 